\title{
Analysis of Biomedical Signals Using Differential Geometry Invariants
}

\author{
F. STUDNIČKA \\ University of Hradec Kralove, Rokitanskeho 62, CZ-500 03 Hradec Kralove, Czech Republic
}

\begin{abstract}
Accelerometers embedded in the medical bed were used to examine the human cardiovascular system. $N$ signals acquired from those sensors were described as 1D manifold and analysed using classical and affine differential geometry invariants. Experiments using elastic tube and two accelerometers were done to model human aorta and to prove the relation presented in the paper of Pinheiro et al.
\end{abstract}

PACS: 47.63.Cb, 87.19.U-

\section{Introduction}

Biomedical signal is a summarizing term for all kinds of signals that can be measured and monitored from human. Unobtrusive measurement of human biosignals is very important in modern medical examination because it can acquire better results in comparison to the classical methods (such as electrocardiography (ECG), blood pressure measurement, applanation tonometry etc.). During the classical measurements, human body is put under stress because the sensors must be somehow placed on the body and e.g. while measuring blood pressure, the arm of a human must be squeezed and the blood pressure (as well as the heart beat rate) can raise significantly. Using applanation tonometry [1] two-pressure sensor has to be sticked on the femoral and carotid artery thus making patient uncomfortable. Several attemps were done to unobtrusively measure human biosignals and to transfer them into relevant markers of the human health [2].

The heart rate can be determined from any biosignal with the same periodicity as the cardiac cycle so ballistocardiographic measurement can be used to determine the heart beat rate. Ballistocardiography (BCG) is a mechanical method for obtaining information about small movements inside the heart (such as the aortic and mitral valve opening/closure). It measures the mass movements of the whole body, generated by the forces of heart contraction and by the blood ejected to large arteries (basically the principle of action and reaction) [3]. Very important marker correlated with the health condition of the person is the heart rate variability (HRV) and the blood pressure variability (BPV). HRV describes the changes in the cardiac cycle period while BPV describes how the blood pressure varies during several cardiac cycle periods.

Human cardiovascular system is a branching graph consisting of aorta, aorta branchings, arteries, etc., on which the pulse wave generated by the heart contraction is propagating (see Fig. 1). There is a strong correlation between the variability of the blood pressure and the pulse wave velocity [4], so time-based experiments were performed by [5]. According to the Moens-Korteweg [6] equation the pulse wave velocity (PWV) is determined by the arterial elasticity and its diameter. If we assume that the elasticity does not change significantly during the experiment, the changes of PWV can be considered being caused by the changes of the aortal blood pressure.

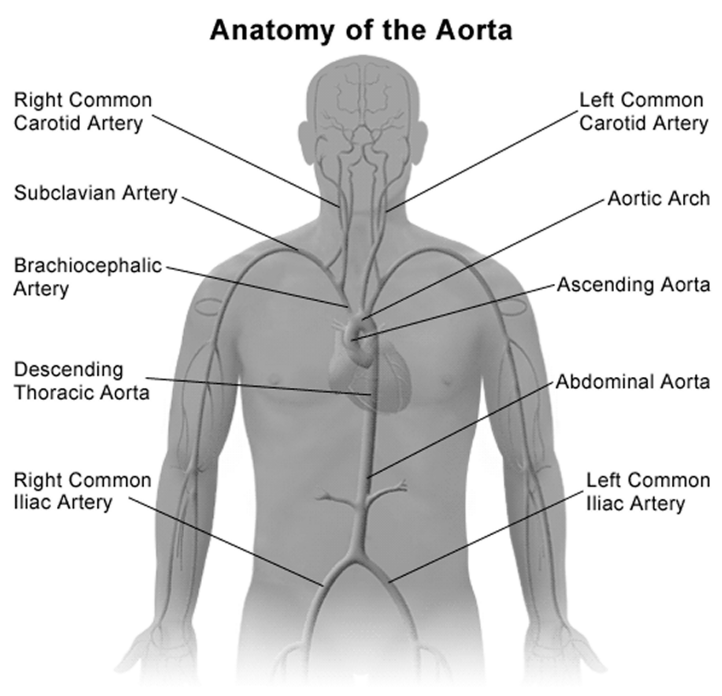

Fig. 1. The shape of human aorta [7].

Relation between the blood pressure and PWV (1) is presented in [5] and [4]:

$$
\mathrm{BP}=a \ln \left(\frac{b}{\left(\frac{d}{P W V}-c\right)^{2}}-1\right),
$$

where BP represents the blood pressure, PWV is the pulse wave velocity and $a, b, c$ and $d$ are constants depen- 
dent on the human physiology. So the relation between the pulse wave velocity and the blood pressure is exponential.

\section{Ballistographic measurement of PWV and HRV}

Ballistographic measurement was done in order to develop completely unobtrusive measurement device which can monitor human HRV and PWV. It is only needed that the patient lies on the bed calmly for a few seconds. If we are able to measure the changes in PWV, it should be possible to transfer those changes into the changes of the blood pressure and thus measure the BPV (see the results of the elastic tube measurement).

The experimental setup for ballistographic measurement consisted of four 3D accelerometers embedded in the legs of the medical bed. More than 50 volunteers were measured on this bed and were also investigated by applanation tonometry, which provides the direct PWV measurement. The ballistographic signal records small movements of the body. Some of those movements are related with the blood ejected from the heart to the aortic arc and also with the pulse wave reflection on the bifurcation in the abdomen (see Fig. 1).

The applanation tonometry is a non-invasive measurement in which two pressure sensors are sticked on the carotid and femoral artery. Using the time delay between the obtained signal it is possible to determine the PWV directly.

\section{Elastic tube measurement}

To check Eq. (1) and to prove that it is experimentally possible to transfer the changes in $\mathrm{PWV}$ into the changes in $\mathrm{BP}$, we modeled the aorta by a $2.3 \mathrm{~m}$ long elastic tube that was placed on the bed.

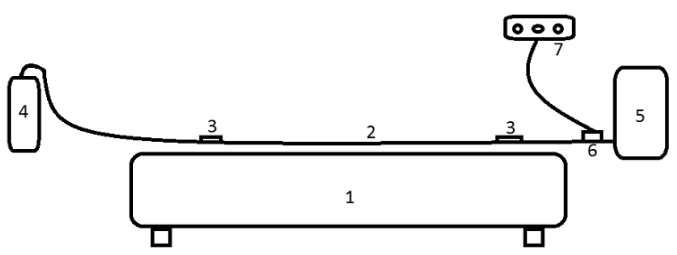

Fig. 2. Scheme of the aorta modelling measurement: 1 - medical bed, 2 - elastic tube, 3 - accelerometers, 4 - water collector, 5 - water container (including pressure pump), 6 - valve, 7 - control unit.

The experimental setup is shown in Fig. 2. On the one end of the elastic tube, a device containing pressure pump, valve, water container and a control unit was attached to represent the human heart. On the second end of tube, large water collector was placed in different heights with respect to the bed to represent the diastolic pressure (see Fig. 2). Two 3D accelerometric sensors were sticked on both ends of the tube.

\section{Results}

All measured data were mathematically analysed using differential geometry invariants. We have acquired 12 signals in the measurement and we treated those 12 signals as a $1 \mathrm{D}$ manifold embedded in $\mathbb{R}^{12}$. Using classical Frenet-Serret theory (see [8] and references therein) we evaluated the arc length and the first two Cartan curvatures. In the elastic tube experiment we used also the affine invariant theory [9] and calculated also the affine arc length.

The classical arc length was calculated using this formula

$$
s(t)=\int_{a}^{b} \sqrt{\sum_{i=1}^{n}\left(\frac{\mathrm{d} C_{i}(t)}{\mathrm{d} t}\right)^{2}} \mathrm{~d} t,
$$

where $s(t)$ is the arc length, $t$ is time, $a$ and $b$ represent the initial and final time for which $s(t)$ is calculated, $i$ is the number of sensors and $C_{i}(t)$ is the signal from $i$-th sensor. To evaluate Cartan curvatures we used a Frenet frame, i.e. the family of orthonormal vectors (the Frenet vectors) $\left\{\boldsymbol{e}_{1}(t), \ldots, \boldsymbol{e}_{n}(t) \mid t \in[a, b]\right\}$ which forms the orthonormal basis related to the curve (see [8] and references therein). The $j$-th curvature can be after that calculated as

$$
c_{j}(t)=\frac{\left\langle\boldsymbol{e}_{j}^{\prime}(t) \cdot \boldsymbol{e}_{j+1}(t)\right\rangle}{\left\|C^{\prime}(t)\right\|}
$$

where $\langle.,$.$\rangle denotes the scalar product in \mathbb{R}^{n}$.

The affine arc length was calculated using a formula, which was derived in [9]:

$$
s(t)=\int[\dot{\gamma}(t), \ddot{\gamma}(t)]^{1 / 3} \mathrm{~d} t,
$$

where $\gamma$ is the $1 \mathrm{D}$ curve embedded in $\mathbb{R}^{n}$ formed by the data from the sensors and [.,.] denotes determinant.

The Euclidean and affine invariant theory was used to study the invariants under the actions of the groups $\mathrm{SO}(3) \times \mathbb{R}^{n}$ and $\mathrm{SL}(3) \times \mathbb{R}^{n}$, respectively. The elements of the first group are invariant under the rotation and translation, this is similar to the human body lying on the bed, since the human cardiovascular system does not change significantly if the patient is lying on his back or on the side, so it is invariant under those transformations. The elements of the second group are invariant under the volume preserving transformations, it means that they are invariant under the transformations that changes shape but preserves collinearity.

For the HRV measurement, the derivative of classical arc length was used to determine peaks with the same periodicity as the cardiac cycle. The HRV is then calculated as the time difference between two adjacent heart beats. For the PWV estimation, we used curvatures reflecting the time delay of the pulse between the aortic arc and the abdominal branching. Finally, for the elastic tube measurement, derivation of the classical and affine arc length was calculated and using peaks therein time delay (and PWV respectively) between the pulse arrival to the sensors was calculated. 


\subsection{HRV measurement}

The comparison of the results using ECG and BCG is shown in Fig. 3. Although the BCG points are slightly more scattered then those from ECG, the error of $\mathrm{BCG}$ is $6.7 \%$ and if we compare the mean values of $\mathrm{HRV}$, we obtain $\left\langle\mathrm{HRV}_{\mathrm{ECG}}\right\rangle=780 \mathrm{~ms}$ and $\left\langle\mathrm{HRV}_{\mathrm{BCG}}\right\rangle=777 \mathrm{~ms}$, which are nearly indentical. The scattering of BCG is mainly produced by the movement of the body (e.g. breathing), by the difference between the electric signal (ECG) and the mechanical response to that signal (BCG), which is not always constant and sometimes by the atypical BCG signal.

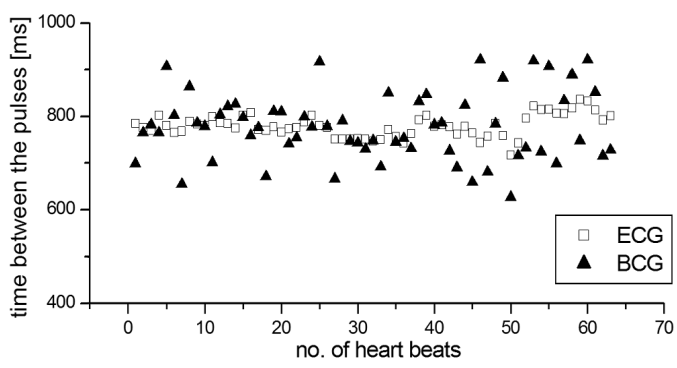

Fig. 3. Sample of HRV obtained by ECG and BCG on one volunteer.

\subsection{Pulse wave velocity measurement}

We compared carotid-femoral time delay measurements using the applanation tonometry with the time-delay by accelerometric sensors in Fig. 4. As time delay we understand the delay between the pulse arrival to some specified points. The accelerometric time delay was measured between the aortic arc and the bifurcation in the abdomen (see Fig. 1). We assume that the distance between the aortic arc and the carotid artery and the distance between the bifurcation and the femoral artery equals. The fitted linear regression is

$$
[\text { acctd }]=0.986[\mathrm{cftd}]+0.747 \text {, }
$$

where [acctd] is accelerometric time delay in $\mathrm{ms}$ and $[\mathrm{cftd}]$ is carotid-femoral time delay in ms.

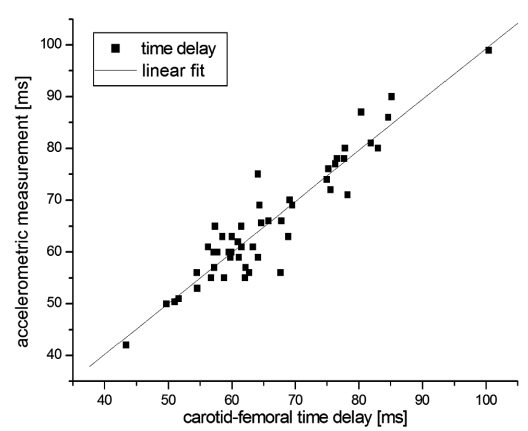

Fig. 4. The values of pulse wave velocity obtained by accelerometric sensors and applanation tonometry.
The Pearson coefficient between these two measurements is 0.938 and the standard deviation is 4.06 , which proves that accelerometric measurement can be used to determine the pulse wave velocity (which can be calculated by estimation of the aortic arc-bifurcation distance).

\subsection{Elastic tube measurement}

Data obtained from the accelerometric sensors sticked to the elastic tube were analysed using the classical and the affine geometry invariants. Both methods provide reasonable results, but the results obtained by the affine invariants were more precise. The dependence of the pulse wave velocity on the water pressure is shown in Fig. 5. The logaritmic scale is used for the pulse wave velocity and the fitted linear regression is

$$
\ln ([\mathrm{pwv}])=1.903[\mathrm{p}]+0.016,
$$

where $[\mathrm{pwv}]$ is pulse wave velocity in $\mathrm{ms}^{-1}$ and $[\mathrm{p}]$ is the pressure in Torr. The Pearson coefficient is 0.984 and the standard deviation is 0.047 . The logarithmic dependence of the pressure on the pulse wave velocity is showing that the formula (1) can be used to determine the blood pressure (and afterwards the blood pressure variability) from the pulse wave velocity, which can be obtained by measuring using accelerometric sensors.

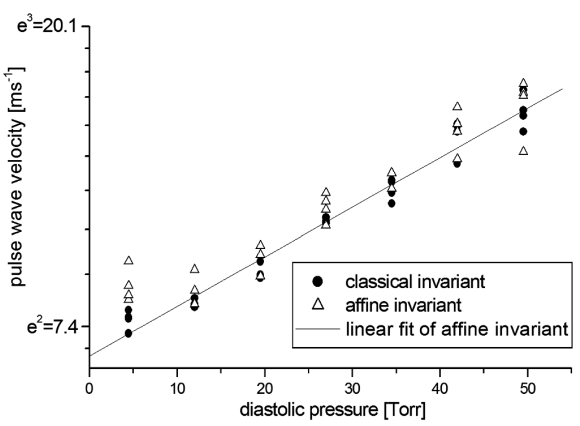

Fig. 5. Measurement of pulse wave velocity propagating along the tube.

\section{Conclusion}

The heart rate variability and the pulse wave velocity were measured using accelerometric sensors embedded in a medical bed. Data from the sensors were analysed using differential geometry invariants. It is shown that the measurement of HRV and the pulse wave velocity using accelerometric sensor is comparable to the standard measurements of those markers. Basic experiments on elastic tube were performed proving an exponential dependence of the pulse wave velocity on the diastolic pressure.

\section{Acknowledgments}

This work was supported by the project LC06002 of the Ministry of Education, Youth and Sports of the Czech 
Republic and the project of the specific research Faculty of Education University of Hradec Kralove, no. 2101/ 2010. The authors are very grateful to Linet, Ltd. company and the Institute for Clinical and Experimental Medicine in Prague. The measurements on the model of aorta were performed by Petr Nezavdal and Cyril Havel.

\section{References}

[1] M.R. Nelson, J. Stepanek, M. Cevette, M. Covalciuc, R.T. Hurst, A.J. Tajik, Mayo Clinic Proc. 85, 460 (2010).

[2] E. Pinheiro, O. Postolache, P. Girão, Open Biomed. Eng. J. 4, 201 (2010).
[3] A.C. Barón, Rev. Colomb. Cardiol. 16, 5 (2009).

[4] Y. Liu, C.C.Y. Poon, Y.-T. Zhang, in: 30th Annual Int. IEEE EMBS Conf., Vancouver (Canada), IEEE 2008, p. 1308.

[5] E. Pinheiro, O. Postolache, P. Girão, Measur. Sci. Rev. 10, 166 (2010).

[6] M.P. Païdoussis, "Fluid-Structure Interactions: Slender Structures and Axial Flow", 2004.

[7] D. Darling, http://www.daviddarling.info/images/ anatomy_of_aorta.gif .

[8] J. Kř́ǐ̌, P. Šeba, Nonlin. Biomed. Phys. 2, 1 (2008).

[9] D. Davis, Ph.D. Thesis, University of Liverpool, 2008. 